\title{
Cryptorchidism is a Useful Clue for Idiopathic Hypogonadotropic Hypogonadism in Pituitary Stalk Thickening
}

\author{
Shamharini Nagaratnam, ${ }^{1}$ Subashini Rajoo, ${ }^{1}$ Mohamed Badrulnizam Long Bidin, ${ }^{1}$ Norzaini Rose Mohd Zain ${ }^{2}$ \\ ${ }^{1}$ Endocrine Unit, Department of Medicine, Hospital Kuala Lumpur, Malaysia \\ ${ }^{2}$ Department of Radiology, Hospital Kuala Lumpur, Malaysia
}

\begin{abstract}
Pituitary stalk lesions can represent a wide range of pathologies. The exact cause is often unknown due to hesitancy to proceed with biopsy. We present a 16-year-old adolescent who presented with delayed puberty, short stature and bilateral cryptorchidism. He was found to have a thickened pituitary stalk of uncertain etiology with partial hypopituitarism (gonadotrophin and growth hormone deficiency) on further assessment. The presence of bilateral cryptorchidism and micropenis represents lack of "mini puberty," a phenomenon of activation of the hypothalamic-pituitary-gonadal (HPG) axis in-utero or within the first few months of life. ${ }^{1}$ These key clinical features have been useful to establish an early temporal relationship and suggest a congenital origin of disease. This enabled a more conservative approach of surveillance to be employed as opposed to invasive pathological examination with pituitary stalk biopsy.
\end{abstract}

Key words: pituitary disease, hypopituitarism, cryptorchidism, growth hormone, hypogonadotrophic hypogonadism

\section{INTRODUCTION}

Pituitary stalk lesions comprise a broad spectrum of diseases that impose a great challenge to the treating physician due to its critical location and pivotal role in the hypothalamic-pituitary (HPA) axis. This proves more of a conundrum in children and adolescents whereby a prompt diagnosis is pertinent to avoid long term repercussions of disease progression and hypopituitarism. The presence of hypopituitarism associated with thickened pituitary stalk implies compromise to the function of the gland which may be permanent or reversible in some cases depending on the etiology and duration of disease. The exact etiology of these lesions often unknown despite rigorous work up, typically warranting a pituitary stalk biopsy as the next step of management. In many cases, there is reluctance to proceed with pathological examination due to the risk of complications. Therefore, the presence of specific clinical features in addition to radiological and laboratory investigations is critical to establish the temporal relationship of insult to the HPA axis and determine possible origin of disease. Bilateral cryptorchidism and micropenis which represents lack of 'mini puberty' are key features supporting an early insult to the hypothalamic-pituitary-gonadal (HPG) axis in patients with hypogonadotrophic hypogonadism. ${ }^{1,2}$

\section{CASE}

We present a 16-year-old boy referred from urology for further evaluation of hypogonadotrophic hypogonadism. He was initially diagnosed with bilateral cryptorchidism aged 2, and his parents opted for conservative management. There was no spontaneous descent of testes in the next few years and he was eventually lost to follow up. He was referred again to urology aged 12, for recurrent urinary tract infections and was diagnosed with vesico-ureteric reflux (VUR) warranting bilateral ureteric stenting and implantation. There were no other structural or functional abnormalities of the renal system identified. Cryptorchidism was reevaluated at this point and he underwent bilateral orchidopexy, with successful descent only on the left side. Routine clinical and hormonal assessment done at this juncture revealed hypogonadotrophic hypogonadism, warranting an endocrine consult.

Detailed history taking from parents and physical examination elicited the following salient clinical features. He was born full-term with an uneventful antenatal history. His height and weight remained within the $25^{\text {th }}$ and $50^{\text {th }}$ centile till the age of 10. A decline in growth rate was noticed as he approached adolescence, whereby he was one of the shortest amongst his peers in secondary school. He had been a slow learner, with poor academic performance since primary school. His parents had noticed him having low energy levels and fatiguability in the later years. No history of anosmia or hearing deficits was elicited. He had no history of polyuria or polydipsia to suggest cranial diabetes insipidus. A full systemic review did not reveal symptoms to suggest intra-cranial mass effect such as chronic headaches, visual disturbances or neurological deficits. He had no past history of intracranial pathology, trauma or radiation. Apart from the history of VUR and cryptorchidism, he did not suffer from any chronic diseases nor receive long term immunosuppression/steroids or chemotherapy. He has no family history of short stature nor delayed puberty. He has one younger sibling, a 12-year-old female who has achieved puberty and is of appropriate height. His anthropometric measurements of weight and height were charted as $58 \mathrm{~kg}$ (between $5-10^{\text {th }}$ 
centile), $155 \mathrm{~cm}$ (below $5^{\text {th }}$ centile) respectively. His height was significantly lower than his mid-parental height of $170 \mathrm{~cm}$. On physical examination, he had no midline facial deformities to suggest a syndromic constellation. Secondary sexual characteristics were also absent. Tanner staging for pubic hair and genitalia was pre pubertal (stage 1) with absent pubic hair, micropenis, minimal hyperpigmentation with no ruggae over the scrotum and testicular volume measuring approximately $1 \mathrm{ml}$ (left) and absent (right).

His anterior pituitary hormone panel showed hypogonadotropic hypogonadism with low FSH, low LH and undetectable testosterone levels. Insulin- like growth factor 1 (IGF-1) levels was found to be lower than expected for his age and gender raising the suspicion of concomitant growth hormone $(\mathrm{GH})$ deficiency. His other pituitary hormones were intact. (Table 1). Insulin Tolerance Test (ITT) with testosterone priming was carried out to confirm growth hormone deficiency. This test was inconclusive due to failure to achieve adequate hypoglycemia.

Table 1. Anterior pituitary hormone panel

\begin{tabular}{|c|c|c|c|c|}
\hline Hormone & \multicolumn{2}{|l|}{ Result } & \multicolumn{2}{|c|}{ Reference range } \\
\hline $\mathrm{FSH}$ & \multicolumn{2}{|l|}{$2.2 \mathrm{IU} / \mathrm{L}$} & \multicolumn{2}{|c|}{$1.5-12.9$} \\
\hline $\mathrm{LH}$ & \multicolumn{2}{|l|}{$0.58 \mathrm{IU} / \mathrm{L}$} & \multicolumn{2}{|c|}{$1.3-9.8$} \\
\hline Testosterone & \multicolumn{2}{|c|}{$<0.1 \mathrm{nmol} / \mathrm{L}$} & \multicolumn{2}{|c|}{$<28.8$} \\
\hline IGF-1 & \multicolumn{2}{|c|}{273 ug/L } & \multicolumn{2}{|c|}{$267-673$} \\
\hline Prolactin & \multicolumn{2}{|c|}{$196.8 \mathrm{mIU} / \mathrm{L}$} & \multicolumn{2}{|c|}{$86-324$} \\
\hline Am cortisol & \multicolumn{2}{|c|}{$569.9 \mathrm{nmol} / \mathrm{L}$} & \multicolumn{2}{|c|}{$145.4-619.4$} \\
\hline \multirow[t]{2}{*}{ Thyroid function test } & $\mathrm{TSH}$ & $2.94 \mathrm{mlU} / \mathrm{L}$ & $\mathrm{TSH}$ & $0.52-4.30$ \\
\hline & Free T4 & $15.76 \mathrm{pmol} / \mathrm{L}$ & T4 & $12.8-21.0$ \\
\hline
\end{tabular}

As an alternative test, Glucagon Stimulation Test (GST) was chosen. GST showed a peak GH of $6.94 \mathrm{ng} / \mathrm{ml}(>10 \mathrm{ng} / \mathrm{ml})$ at 90 mins confirming his growth hormone deficiency. ${ }^{3}$ Bone age assessment showed his skeletal age to lag at the age of 12-13 years with unfused epiphyseal plates. A MRI pituitary ordered showed an enlarged pituitary stalk extending to the tuber cinereum with homogenous enhancement post gadolinium contrast. The stalk measured $13 \mathrm{~mm}$ (AP diameter) at the point of insertion at the infundibulum with uniformed pattern of thickening. The pituitary gland itself was not hypoplastic, with no focal enhancement on post contrast study. The optic nerve, chiasm and tract was not thickened, with no evidence of compression. (Figures 1 and 2). Therefore, at this point it was concluded that this patient had thickened pituitary stalk with partial hypopituitarism (hypogonadotropic hypogonadism and growth hormone deficiency).

The etiology of thickened pituitary stalk was worked up extensively involving laboratory and radiological investigations. His complete blood count showed no evidence of hematological dyscrasias. His liver and renal biochemistry was normal. Both inflammatory and tumor markers were not raised. There was no evidence of mediastinal or lung mass on chest x-ray. Ultrasound showed very small testes in the right inguinal region and left scrotum measuring $1.2-1.5 \mathrm{~cm}$, both testes had no suspicious malignant features or associated lymphadenopathy. In summary, there were no red flags to suggest an inflammatory or neoplastic process. A multidisciplinary discussion was held to discuss the role of pituitary stalk biopsy for him. The presence of pre-pubertal features

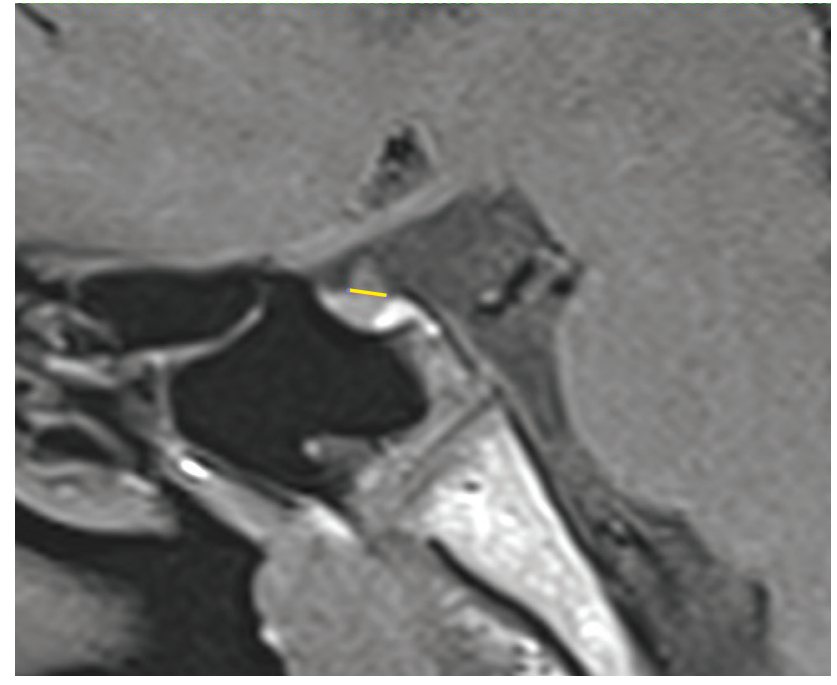

Figure 1. Sagittal T1WI MRI Pituitary showing enlargement of the pituitary stalk, measuring $13 \mathrm{~mm}$ in AP diameter.

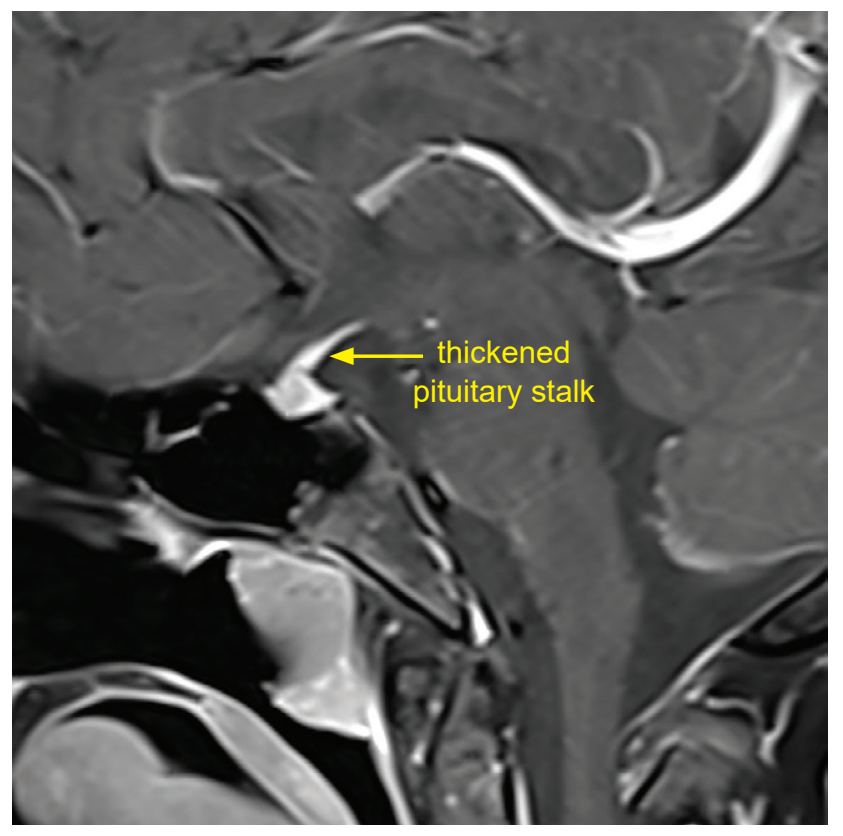

Figure 2. Post gadolinium sagittal (T1WI) MRI of pituitary showing homogenous enhancement of the pituitary stalk (yellow arrow). The pituitary gland is small in size and has no focal lesion.

representing gonadotroph deficiency early in life coupled with non-suspicious laboratory and imaging workup was highly suggestive of congenital origin of disease. Therefore, a decision was made not to proceed with pituitary stalk biopsy unless there is evidence of disease extension or progressive loss of pituitary function.

Growth hormone, norditrophin at a dose of $0.025 \mathrm{mcg} / \mathrm{kg} /$ day was initiated in this boy aiming to achieve near adult height. He showed good response to therapy with a $4 \mathrm{~cm}$ increment in height after 3 months. Puberty induction will be initiated once acceptable near adult height is achieved. Serial MRI pituitary and pituitary hormone panel will be repeated every 6 months to monitor progression of disease. He is also planned for removal of the right testis due to risk of malignancy and poor function. 


\section{DISCUSSION}

The etiology of thickened pituitary stalks is often broadly divided into 3 categories: neoplastic, inflammatory or infectious and congenital. ${ }^{4}$ Two large case series based on retrospective review involving both adults and children with pituitary stalk lesions have showed neoplastic lesions to be the leading cause of pathology.,5 A similar finding was found in a study done in Korea, specifically in the pediatric population below the age of 18 with pituitary stalk lesions. ${ }^{6}$ However, Turcu et al., also found 39\% of pituitary stalk lesions in a retrospective review with unclear pathology. ${ }^{4}$ The role of pituitary stalk biopsy often remains controversial due to the risk involved. Despite using a minimally invasive endoscopic approach, the procedure, coupled with manipulation of the pituitary stalk, imposes a risk of cranial diabetes insipidus, hypopituitarism, cerebrospinal fluid (CSF) leak and meningitis in patients. In addition, there is approximately a $10 \%$ risk of a negative biopsy, whereby a diagnosis is not histologically conclusive despite adequate tissue sample. ${ }^{7}$

Radiological features on MRI is often the key in guiding the diagnosis of pituitary stalk lesions. It is also an essential tool to monitor progression of disease or response to treatment in these patients. Specific patterns of enhancement such as uniform, V-shaped, round or diamond, and pyramidal has been associated with various pathologies in literature. The strongest association found was between the congenital lesions and round pattern of enhancement in the Mayo experience by Turcu et al. Extent of lesions was also associated with hypopituitarism, and the lesions with hypothalamic extension have been found to have the highest risk of hypopituitarism. ${ }^{4}$

Congenital cryptorchidism is one of the most common congenital malformation in boys. Its prevalence at birth among boys with a birth weight more than 2,500 $\mathrm{g}$ has been reported to range between $1.8 \%$ and $8.4 \%$. It is associated with reduced concentration of testosterone and sperm quality in adulthood. There are numerous causes for congenital cryptorchidism including disorders involving sex chromosomes, gonadal development, decrease in androgen synthesis and action, structural defects and hypogonadotrophic hypogonadism.

Typically, pituitary gonadotropes start producing FSH and LH around 9 weeks of gestation, reaching their peaks at mid-gestation with a concomitant rise in testosterone. This peak of testosterone is essential for descent of testes in utero. The activation of hypothalmic-pituitary-gonadal (HPG) axis occurs approximately 1 week after birth with a second peak at 1-3 months, a period also synonymous with 'mini-puberty.' This post- natal surge is essential for testicular descent and penile growth. In those with congenital hypogonadotropic hypogonadism, the lack of gonadotrophins results in the arrest of testicular descent and penile growth in utero and in the neonatal period. ${ }^{2}$

\section{CONCLUSION}

In our patient, the lack of 'mini puberty' described above suggests the insult to the HPG axis occurred early in life, pointing towards a congenital origin of disease. Despite the radiological features not being typically described in literature for congenital lesions, the extension of the lesion to the hypothalamus accounts for the development of partial hypopituitarism in this patient. In addition, the negative results for both inflammatory and neoplasm workup further supports this diagnosis. With congenital disease as our probable diagnosis, we have chosen a more conservative approach of management with surveillance as described above, alleviating the need for pituitary stalk biopsy at the moment.

Ethical Consideration

Patient consent was obtained before submission of the manuscript.

Statement of Authorship

All authors certified fulfilment of ICMJE authorship criteria.

\section{Author Disclosure}

The authors declared no conflict of interest.

\section{Funding Source}

None.

\section{References}

1. Boehm U, Bouloux P, Dattani MT, et al. Expert consensus document: European Consensus Statement on congenital hypogonadotropic hypogonadism--pathogenesis, diagnosis and treatment. Nat Rev Endocrinol. 2015;11(9):547-64. PMID: 26194704. https://doi.org/10.1038/ nrendo.2015.112.

2. Rodprasert W, Virtanen HE, Mäkelä JA, Toppari J. Hypogonadism and cryptorchidism. Front Endocrinol (Lausanne). 2020;10:906. PMID: 32010061. PMCID: PMC6974459. https://doi.org/ 10.3389/ fendo.2019.00906.

3. Growth Hormone Research Society. Consensus guidelines for the diagnosis and treatment of growth hormone $(\mathrm{GH})$ deficiency in childhood and adolescence: Summary statement of the GH Research Society. J Clin Endocrinol Metab. 2000;85(11):3990-3. PMID: 11095419. https://doi.org/10.1210/jcem.85.11.6984.

4. Turcu AF, Erickson BJ, Lin E, et al. Pituitary stalk lesions : The Mayo Clinic experience. J Clin Endocrinol Metab. 2013;98(5):1812-8. PMID 23533231. https://doi.org/10.1210/jc.2012-4171.

5. Hamilton BE, Salzman KL, Osborn AG, Be H, Kl S, Ag O. Anatomic and pathologic spectrum of pituitary infundibulum lesions. AJR Am J Roentgenol. 2007;188(3):223-32. PMID: 17312027. https://doi. org/10.2214/AJR.05.2027.

6. Yoon SC, Shin HS, Yang SW, Lee SY. Clinical and radiological features of pituitary stalk lesions in children and adolescents. Ann Pediatr Endocrinol Metab. 2014;19(4):202-7. PMID: 25654066. PMCID: PMC4316407. https://doi.org/10.6065/apem.2014.19.4.202.

7. Catford S, Wang YY, Wong R. Pituitary stalk lesions: Systematic review and clinical guidance. Clin Endocrinol (Oxf). 2016;85(4):507-52. PMID 26950774. https://doi.org/10.1111/cen.13058.

Authors are required to accomplish, sign and submit scanned copies of the JAFES Author Form consisting of: (1) Authorship Certification, that authors contributed substantially to the work, that the manuscript has been read and approved by all authors, and that the requirements for authorship have been met by each author; (2) the Author Declaration, that the article represents original material that is not being considered for publication or has not been published or accepted for publication elsewhere, that the article does not infringe or violate any copyrights or intellectual property rights, and that no references have been made to predatory/suspected predatory journals; (3) the Author Contribution Disclosure, which lists the specific contributions of authors; (4) the Author Publishing Agreement which retains author copyright, grants publishing and distribution rights to JAFES, and allows JAFES to apply and enforce an Attribution-Non-Commercial Creative Commons user license; and (5) the Conversion to Visual Abstracts (*optional for original articles only) to improve dissemination to practitioners and lay readers Authors are also required to accomplish, sign, and submit the signed ICMJE form for Disclosure of Potential Conflicts of Interest. For original articles, authors are required to submit a scanned copy of the Ethics Review Approval of their research as well as registration in trial registries as appropriate. For manuscripts reporting data from studies involving animals, authors are required to submit a scanned copy of the Institutional Animal Care and Use Committee approval. For Case Reports or Series, and Images in Endocrinology, consent forms, are required for the publication of information about patients; otherwise, appropriate ethical clearance has been obtained from the institutional review board. Articles and any other material published in the JAFES represent the work of the author(s) and should not be construed to reflect the opinions of the Editors or the Publisher. 